Research Article

\title{
Iranian Pediatric COVID-19 Epidemiology and Clinical Characteristics
}

\author{
Shahnaz Armin (D), 1 Mohammadreza Mirkarimi $\mathbb{D}^{1},{ }^{2}$ Zahra Pourmoghaddas $\left(\mathrm{D},{ }^{3}\right.$ \\ Marjan Tariverdi $\mathbb{D},{ }^{4}$ Azadeh Jafrasteh $\mathbb{D},{ }^{5}$ Noushin Marhamati $\mathbb{D}^{1},{ }^{1}$ Armin Shirvani $\mathbb{D},{ }^{6}$ \\ Abdollah Karimi $\mathbb{D}^{1}$, Sedigheh Rafiei Tabatabaei $\mathbb{D}^{1},{ }^{1}$ Roxana Mansour Ghanaei $\mathbb{D}^{1}{ }^{1}$ \\ SeyedAlireza Fahimzad $\mathbb{D}^{\mathbb{D}},{ }^{1}$ Fariba Shirvani $\mathbb{D}^{1},{ }^{1}$ and Seyedeh Mahsan Hoseini-Alfatemi $\mathbb{B}^{1}$ \\ ${ }^{1}$ Pediatric Infections Research Center, Research Institute for Children's Health, Shahid Beheshti University of Medical Sciences, \\ Tehran, Iran \\ ${ }^{2}$ Aboozar Children's Medical Center, Ahvaz Jundishapur University of Medical Sciences, Ahvaz, Iran \\ ${ }^{3}$ Pediatrics Infectious Disease Department, Isfahan University of Medical Sciences, Isfahan, Iran \\ ${ }^{4}$ Department of Pediatric, Clinical Research Development Center of Children Hospital, \\ Hormozgan University of Medical Sciences, Bandar Abbas, Iran \\ ${ }^{5}$ Department of Pediatrics, School of Medicine, Lorestan University of Medical Sciences, Khorramabad, Iran \\ ${ }^{6}$ Virtual School of Medical Education and Management, Shahid Beheshti University of Medical Sciences, Tehran, Iran
}

Correspondence should be addressed to Seyedeh Mahsan Hoseini-Alfatemi; mahsan.hoseinialfatemi@gmail.com

Received 8 September 2021; Accepted 25 November 2021; Published 15 December 2021

Academic Editor: Louis Detolla

Copyright (c) 2021 Shahnaz Armin et al. This is an open access article distributed under the Creative Commons Attribution License, which permits unrestricted use, distribution, and reproduction in any medium, provided the original work is properly cited.

\begin{abstract}
Background. Despite the worldwide spread of Severe Acute Respiratory Syndrome Corona Virus 2 (SARS-CoV-2), information about the epidemiological and clinical patterns of this infection is still largely unknown in children. In addition, the prevalence of this disease is still very high in some parts of the world, including Iran. Thus, this study aims to evaluate the epidemiological features, laboratory and imaging findings, and the type of treatments in children with novel coronavirus 2019 (COVID-19). Method. This study is conducted from March 2020-March 2021 by using the medical records of hospitalized confirmed COVID19 children younger than 18 years in five cities of Iran: Tehran, Ahwaz, Isfahan, Bandar-Abbas, and Khorramabad. In addition to demographic and epidemiological data, we also studied clinical signs and treatments. Results. In total 278 confirmed COVID-19 children, the average age was 5.3 years, and $59.4 \%$ were boys. A total of $37.8 \%$ had an underlying disease, in which the most common was a malignancy. The most common symptoms were fever and cough. In this group of pediatrics, some abnormal laboratory findings have been seen. GGO (Ground-Glass Opacity) had been diagnosed in $58.6 \%$ of children. 3.6\% needed oxygen therapy with ventilators, and $83.09 \%$ had received antibiotic treatments with the majority of ceftriaxone. Also, $10 \%$ had got steroids. In this study, the mortality rate was $4.3 \%$. Conclusion. In this study, most of the children who died had an underlying disease, so timely care and action is important in them. Most children admitted to our study received antibiotics and were prescribed antivirals and steroids for a smaller number. Also, a small number of children received oxygen therapy, most of whom were in the age group of 1 to 5 years.
\end{abstract}

\section{Introduction}

Coronavirus disease 2019 (COVID-19) is caused by severe acute respiratory syndrome coronavirus-2 (SARS-CoV-2). Because of the intensity and alarming level of spread, the World Health Organization (WHO) deemed it a pandemic
[1]. The first pandemic infection to occur due to a coronavirus [2], SARS-CoV-2, is a factor that effects all age groups.

COVID-19 has a low incidence of severe cases among children. Children have a milder clinical course than adults [3-6]. Children and adults have different levels of immune 
maturity, which may be the reason for differences in the prevalence and type of clinical manifestations of COVID-19 [7]. However, it is also important to note that even mild COVID-19 can cause transmission [8]. According to the work of Gandhi et al., asymptomatic transmission is the Achilles' heel of this pandemic [9].

In other words, children can cause cluster propagation in the home environment [10]. Furthermore, children with gastrointestinal symptoms can transmit the virus through their feces for weeks, which is dangerous in some places such as kindergartens or elementary schools [11-13].

In addition to acute COVID-19 in children, there are several reports of multisystemic involvement, which complicates the diagnosis and management of this disease in pediatrics.

First cases of COVID-19 were observed in Iran in January 2020, but the country began reporting cases to the WHO on February 2020 [14]. Since then, the disease has spread rapidly across the country, infecting many children [5]. The purpose of this study was to determine the clinical presentation and treatment pattern of COVID-19 among children admitted to 5 major cities in Iran.

\section{Method}

We conducted a retrospective, cross-sectional, multicenter study from March 2020 to March 2021 in several Iranian cities (Tehran, Ahwaz, Isfahan, Bandar-Abbas, and Khorramabad). During the study period, all children hospitalized with confirmed SARS-CoV-2 infection in one of the participating centers were included. Data were extracted from medical records of children younger than 18 years diagnosed with confirmed cases of COVID-19 with positive real-time reverse transcription polymerase chain reaction (RT-PCR) results on nasopharyngeal samples. Besides demographic and epidemiological data, we also studied clinical symptoms and treatments. Data were collected from medical forms and records by a pediatrician. No written consent has been obtained from the patients as there are no patient identifiable data included in this research.

The research ethics committee of Shahid Beheshti University of Medical Sciences approved the study (Ethical code: IR.SBMU.RICH.REC.1400.007).

\section{Result}

The average age of the 278 children and adolescents hospitalized with COVID-19 was 5.3 years. Of the 278 patients, $56(20.1 \%)$ were under one year of age, $95(34.2 \%)$ were between 1 and 5 years of age, and 127 (45.7\%) were over five years of age. There were $59.4 \%$ boys among the cases. Results of the study, which include general information and clinical signs, are divided into 3 age groups (under 1 year, 1 to 5 years, and over 5 years) in Figure 1 .

A total of $37.8 \%$ of children hospitalized had an underlying disease. By far, the most common condition was malignancy (10\%), followed by diabetes (5\%). We estimate that the mortality rate was $4.3 \%$ (12 patients) as 8 of them had an underlying disease, and half of the dead were over 5

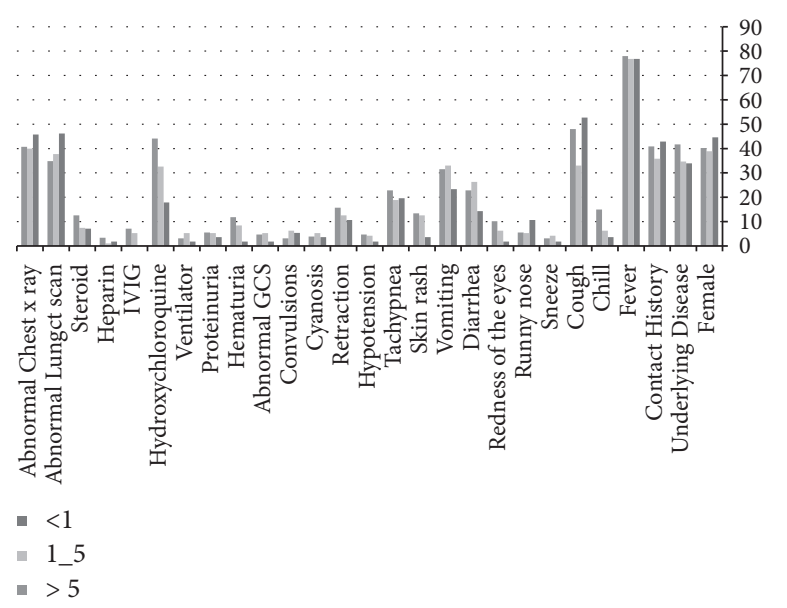

FIGURE 1: General information and clinical symptoms of pediatric patients with confirmed COVID-19 in 3 age groups $(<1,1-5,>5)$.

years of age. Approximately $40 \%$ of our study population was exposed to an indicator case or an adult with suspected SARS-CoV-2 infection. 3.6\% of hospitalized children needed oxygen therapy with ventilators, most of whom were 1 to 5 years old.

Children with COVID-19 tend to experience fewer symptoms than adults. Fever was the most common symptom (77.3\%), according to the information obtained. Cough was another symptom (43.8\%). Both diarrhea and vomiting were present in $22.3 \%$ and $30.3 \%$ of cases, respectively. In $20.9 \%$ of cases, tachypnea was reported. Infection was also associated with sneezing and runny nose in $3.2 \%$ and $6.5 \%$ of children, respectively. A skin rash was reported in $11.2 \%$ of children hospitalized with COVID-19 and red eyes in $7.2 \%$. The mean O2 level was 93.6\% (with a minimum of 68 and a maximum of $99 \%$ ). $\mathrm{O}_{2}$ was below $93 \%$ in $22.6 \%$ of cases. Frequently, children hospitalized for illnesses have elevated inflammatory markers, such as ESR, CRP, and liver enzyme. The mean initial CRP in the under one age group was lower than in other age groups. BUN and Cr were abnormal in 13.6\% and $8 \%$ patients, respectively. In our research, based on the division of LDH into two groups above 500 and above 1000 , patients were included in 56.88 and $8.3 \%$, respectively. Abnormal AST and ALT were found in $37.17 \%$ and $15.03 \%$, respectively. Laboratory findings are presented in Table 1. In our review, leukocytosis had been reported in $24.5 \%$ of the patients, while $9.33 \%$ of our pediatrics had leukopenia. Different age groups have reported lymphopenia: $44 \%$ under one year of age, $28.04 \%$ in 1 to 5 years, and $34.25 \%$ in people 5 years and older. GGO has been diagnosed in $58.6 \%$ of children with lung involvement, mainly in RLL and LLL.

A total of 231 patients $(83.09 \%)$ were treated with antibiotics, with the most common drug used being ceftriaxone (161), followed by vancomycin (67), meropenem (48), and azithromycin (42), given as a two- or three-medicine regimen. There were also 100 patients given antivirals (35.97\%), most of whom were given Kaletra (52 patients) and atazanavir (22 patients). Also 10\% had steroid treatments. 
TABLE 1: Laboratory findings of the hospitalized COVID-19 pediatric patients.

\begin{tabular}{|c|c|c|c|c|c|}
\hline Laboratory data & Number & Minimum & Maximum & Mean & Std. deviation \\
\hline $\mathrm{O}_{2}$ sat in room temperature & 137 & 68 & 99 & 93.66 & 5.379 \\
\hline WBC & 257 & 120 & 43600 & 8979.96 & 5415.383 \\
\hline ALC & 242 & 86 & 16947 & 3063.30 & 2527.806 \\
\hline $\mathrm{Hb}$ & 257 & 4.50 & 17.70 & 11.12 & 2.068 \\
\hline PLT & 256 & 6000 & 838000 & 271807.46 & 144975.231 \\
\hline CRP & 208 & 0 & 178 & 19.28 & 27.452 \\
\hline ESR & 226 & 1 & 210.7 & 26.90 & 27.78 \\
\hline BS & 201 & 1 & 891.5 & 124.93 & 96.95 \\
\hline BUN & 249 & 3 & 1542 & 24.11 & 110.221 \\
\hline $\mathrm{Cr}$ & 249 & 0.10 & 54 & 1.04 & 3.584 \\
\hline SGOT & 155 & 5 & 232 & 42.62 & 31.750 \\
\hline SGPT & 152 & 3 & 1478 & 36.53 & 119.919 \\
\hline ALK.P & 103 & 93 & 1822 & 477.08 & 299.025 \\
\hline PT & 78 & 10 & 32 & 13.70 & 4.114 \\
\hline PTT & 74 & 12.20 & 70 & 32.74 & 9.504 \\
\hline INR & 77 & 0.78 & 1.50 & 1.07 & 0.139 \\
\hline $\mathrm{LDH}$ & 167 & 6.90 & 2697 & 623.62 & 387.434 \\
\hline Ferritin & 65 & 4 & 1714 & 311.25 & 386.216 \\
\hline Fibrinogen & 20 & 67 & 1000 & 330.30 & 196.052 \\
\hline $\mathrm{CPK}$ & 113 & 16 & 2126 & 155.53 & 230.546 \\
\hline D.dimer & 45 & 0.09 & 3327 & 581.64 & 776.268 \\
\hline Troponin & 53 & 0 & 28.80 & 5.03 & 6.903 \\
\hline $\mathrm{PH}$ & 81 & 6.91 & 7.70 & 7.34 & 0.143 \\
\hline $\mathrm{PCO} 2$ & 81 & 14 & 98 & 35.53 & 12.199 \\
\hline $\mathrm{HCO} 3$ & 80 & 5.70 & 27.70 & 19.35 & 4.323 \\
\hline
\end{tabular}

\section{Discussion}

In Wenjun et al.'s retrospective study, the median age was 6.2 years [15], while in ours, the mean age was 5.3 years. The study showed that boys made up the majority (59.4\%), as in other studies including the work of Dong et al. (56.6\%) [5]. Hua et al. in an epidemiological study reported the mean age in children was 8.16 years (in the range of 3.66 months-14 years) and $60.5 \%$ were male [16].

In the study by Tezer and Bedir Demirdağ, among 345 confirmed children, $23 \%$ had underlying diseases and the most common ones were chronic pulmonary disease (counting asthma), cardiovascular disease, and immunosuppression (caused by cancer, chemotherapy, etc.) [17]. In addition, in Kompaniyets et al.'s cross-sectional study, among 43,465 patients with COVID-19 pediatrics, $28.7 \%$ had underlying medical conditions, mostly asthma, neurodevelopmental disorders, anxiety-related disorders, depressive disorders, and obesity [18]. Also, we found that $37.8 \%$ of our hospitalized children had an underlying disease with the most common being malignancy followed by a chronic disease such as diabetes. The differences likely arise from the type of underlying conditions that researchers are searching for.

Hoang et al.'s study had reported 0.09\% deaths among the COVID-19 children [19], while by our results, the estimated mortality rate was below 5 percent.

According to Chang et al.'s systematic literature (with last updates on 15 March 2020), as well as Ansel et al.'s systematic search (with last searched 14 May 2020) and Hoseinyazdi et al.'s pediatric study in Shiraz (March-May 2020), like ours, fever and cough were the most common clinical symptoms [19-21]. Although Chang et al.'s study reported few gastrointestinal symptoms (12\%) [20], in our study, diarrhea and vomiting were reported in $22.3 \%$ and $30.3 \%$ of cases, respectively. Possibly because we studied at a different time and place, the predominant virus strain may have been different.

Two systematic reviews have shown that most children with COVID-19 have a normal WBC count and the most common abnormality is leukopenia $[22,23]$; this variance may be due to differences in age group or severity of the infection or virus.

In our review, $24.5 \%$ of the patients had leukocytosis, while only $9.33 \%$ had shown leukopenia. In a systematic review by Henry et al., leukocyte counts were normal in most children, and lymphopenia was present in only $3 \%$, none of whom had severe disease [24].

In our investigations, out of 240 patients, $34.16 \%$ had been shown with lymphopenia and only $2 \%$ had lymphocytosis. Based on Hoseinyazdi et al.'s pediatric study in Namazi and Ali-Asghar Hospitals, lymphocytosis has been shown in severe cases [21]. Additionally, another study by $\mathrm{Du}$ et al. in Shandong Province in China found increased lymphocyte counts in children compared to adults [15]. According to Kosmeri et al., lymphocytosis was the most common findings in neonates and infants with COVID-19. Moreover, anemia and thrombocytopenia were rarely seen in COVID-19 children [25].

In Hoseinyazdi's study, CRP levels were normal in patients under two years of age, whereas they were significantly higher in those aged over 3 years [21]. Also, in our study, the mean range of initial CRP was lower in the group under 1 years of age than that of those over this age.

Abnormal levels of $\mathrm{LDH}$ were seen in fifty percent of children with COVID-19 in the study by Du et al. and also 
showed that positive LDH levels were significantly higher in children than in adults [15]. In ours, more than half of the study population had an $\mathrm{LDH}$ above 500 and less than a tenth had it above 1000. Furthermore $37.17 \%$ and $15.03 \%$ had abnormal AST and ALT in that order; while in Esmaeili et al.'s retrospective study, $27.8 \%$ and $38.9 \%$ of cases showed abnormally high ALT and AST levels, respectively [26].

According to an Italian report from an emergency department for children, $4 \%$ of children had oxygen saturation below 95\%. All of these patients also had imaging evidence of lung involvement [4]. In our observations, the mean $\mathrm{O}_{2}$ saturation level was $93.66 \%$ and $\mathrm{O}_{2}$ sat was below $93 \%$ in $22.6 \%$ of reported cases. Since our data pertain to sick patients admitted to hospitals, the figure is higher than Italian rates, even though most Italian patients had not been in a bad situation.

The most common radiographic finding in Chang et al.'s investigations was ground-glass opacities (48\%) [20]. In Samy and Khalaf's investigations, 44 (83\%) patients had normal CT and only 9 patients presented lung opacities in which 5 cases showed consolidation and 2 cases were with GGO, while in another 2 cases, consolidation with GGO was noted. The most involved lobes were the right and left lower lobes [27]. In our study, GGO was the most commonly reported finding in $58.6 \%$ of children with pulmonary involvement (in RLL and LLL).

In one of the observational studies in Wuhan, China, six of the eight patients received high-flow oxygen therapy and two critically ill patients were mechanically ventilated. Antiviral therapies (Verazole, oseltamivir, and interferon) were administered to all patients. Antibiotics (in 5/8), traditional Chinese medicine (in $4 / 8$ ), intravenous immunoglobulins (in 4/8), and glucocorticoids (in 5/8) were also used according to the children's condition [28].

In our education, a total of 231 patients $(83.09 \%)$ received antibiotic treatment, the majority of which were ceftriaxone. vancomycin, meropenem, and azithromycin; on the other hand, they were also commonly administered in combination with each other. A total of 100 patients were also prescribed antiviral medicines, the majority of whom took Kaletra or atazanavir based on national protocols at the time of the study. In addition, about $10 \%$ of our patients received steroid medication. Fewer than $4 \%$ of hospitalized children required respiratory oxygen therapy, most of whom were between the ages of 1 and 5 years.

Based on a retrospective study by Zhang et al., ribavirin was given to $44 \%$ of patients. $85 \%$ had received antibiotic therapy. Corticosteroids (15\%) and supportive oxygen inhalation therapy (9\%) were also used [29].

\section{Conclusions}

Among the children who died in this study, the majority had the comorbidities. In order to protect children with underlying diseases, care and isolation must be provided in a timely manner. Cough and fever were the most common clinical symptoms. Leukocyte changes, especially lymphopenia, have been reported less frequently in children under 1 year of age with COVID-19, possibly due to their immature immune systems and ACE2 expression. Most patients received antibiotics, and relatively fewer antivirals and steroids were administered. Also, oxygen therapy was used to a much lesser extent in our patients, most of whom were in the age group of 1-5 years. Therefore, paying attention to the abovementioned results can help us reduce the prevalence of this disease.

\section{Data Availability}

The authors declare the data used to support the findings of this study are available from the corresponding author upon request.

\section{Additional Points}

Because this is a retrospective study that collected information from medical records in different cities, some data and variables were not available; in addition, it was not possible to complete it (for example, some tests were not performed in all patients).

\section{Conflicts of Interest}

The authors declare no conflicts of interest.

\section{References}

[1] E. Livingston and K. Bucher, "Coronavirus disease 2019 (COVID-19) in Italy," Journal of the American Medical Association, vol. 323, no. 14, p. 1335, 2020.

[2] WHO, Coronavirus Disease (COVID-19), WHO, Geneva, Switzerland, 2020

[3] X. Lu, L. Zhang, H. Du et al., "SARS-CoV-2 infection in children," New England Journal of Medicine, vol. 382, no. 17, pp. 1663-1665, 2020.

[4] N. Parri, M. Lenge, and D. Buonsenso, "Children with covid19 in pediatric emergency departments in Italy," New England Journal of Medicine, vol. 383, no. 2, pp. 187-190, 2020.

[5] Y. Dong, X. Mo, Y. Hu et al., "Epidemiology of COVID-19 among children in China," Pediatrics, vol. 145, no. 6, 2020.

[6] CDC COVID-19 Response Team, "Coronavirus disease 2019 in children-United States, february 12-april 2, 2020," Morbidity \& Mortality Weekly Report, vol. 69, pp. 422-426, 2020.

[7] D. Raoult, A. Zumla, F. Locatelli, G. Ippolito, and G. Kroemer, "Coronavirus infections: epidemiological, clinical and immunological features and hypotheses," Cell Stress, vol. 4, no. 4, pp. 66-75, 2020.

[8] W.-j. Guan, Z.-y. Ni, Y. Hu et al., "Clinical characteristics of coronavirus disease 2019 in China," New England Journal of Medicine, vol. 382, no. 18, pp. 1708-1720, 2020.

[9] M. Gandhi, D. S. Yokoe, and D. V. Havlir, "Asymptomatic transmission, the Achilles' heel of current strategies to control covid-19," New England Journal of Medicine, vol. 382, no. 22, pp. 2158-2160, 2020.

[10] "Questions and answers on COVID-19: children aged 1-18 years and the role of school settings," 2020, https://www.ecdc. europa.eu/en/covid-19/questions-answers/questionsanswers-school-transmission.

[11] Y. Xu, X. Li, B. Zhu et al., "Characteristics of pediatric SARSCoV-2 infection and potential evidence for persistent fecal 
viral shedding," Nature Medicine, vol. 26, no. 4, pp. 502-505, 2020.

[12] X. Ma, L. Su, Y. Zhang, X. Zhang, Z. Gai, and Z. Zhang, "Do children need a longer time to shed SARS-CoV-2 in stool than adults?" Journal of Microbiology, Immunology, and Infection, vol. 53, no. 3, pp. 373-376, 2020.

[13] Y.-H. Xing, W. Ni, Q. Wu et al., "Prolonged viral shedding in feces of pediatric patients with coronavirus disease 2019," Journal of Microbiology, Immunology, and Infection, vol. 53, no. 3, pp. 473-480, 2020.

[14] World Health Organization, Coronavirus Disease (COVID2019) Situation reportsWorld Health Organization, Geneva, Switzerland, 2020.

[15] W. Du, J. Yu, H. Wang et al., "Clinical characteristics of COVID-19 in children compared with adults in Shandong Province, China," Infection, vol. 48, no. 3, pp. 445-452, 2020.

[16] C. Z. Hua, Z. P. Miao, J. S. Zheng et al., "Epidemiological features and viral shedding in children with SARS-CoV-2 infection," Journal of Medical Virology, vol. 92, no. 11, pp. 2804-2812, 2020.

[17] H. Tezer and T. Bedir Demirdağ, "Novel coronavirus disease (COVID-19) in children," Turkish Journal of Medical Sciences, vol. 50, no. SI-1, pp. 592-603, 2020.

[18] L. Kompaniyets, N. T. Agathis, J. M. Nelson et al., "Underlying medical conditions associated with severe COVID-19 illness among children," JAMA Network Open, vol. 4, no. 6, pp. e2111182-e, 2021.

[19] A. Hoang, K. Chorath, A. Moreira et al., "COVID-19 in 7780 pediatric patients: a systematic review," EClinicalMedicine, vol. 24, Article ID 100433, 2020.

[20] T.-H. Chang, J.-L. Wu, and L.-Y. Chang, "Clinical characteristics and diagnostic challenges of pediatric COVID-19: a systematic review and meta-analysis," Journal of the Formosan Medical Association, vol. 119, no. 5, pp. 982-989, 2020.

[21] M. Hoseinyazdi, S. Esmaeilian, R. Jahankhah et al., "Clinical, laboratory, and chest CT features of severe versus non-severe pediatric patients with COVID-19 infection among different age groups," BMC Infectious Diseases, vol. 21, no. 1, pp. 560-612, 2021.

[22] J. Meena, J. Yadav, L. Saini, A. Yadav, and J. Kumar, "Clinical features and outcome of SARS-CoV-2 infection in children: a systematic review and meta-analysis," Indian Pediatrics, vol. 57 , no. 9, pp. 820-826, 2020.

[23] N. A. Patel, "Pediatric COVID-19: systematic review of the literature," American Journal of Otolaryngology, vol. 41, no. 5, Article ID 102573, 2020.

[24] B. M. Henry, G. Lippi, and M. Plebani, "Laboratory abnormalities in children with novel coronavirus disease 2019," Clinical Chemistry and Laboratory Medicine, vol. 58, no. 7, pp. 1135-1138, 2020.

[25] C. Kosmeri, E. Koumpis, S. Tsabouri, E. Siomou, and A. Makis, "Hematological manifestations of SARS-CoV-2 in children," Pediatric Blood \& Cancer, vol. 67, no. 12, Article ID e28745, 2020.

[26] M. Esmaeili Dooki, S. Mehrabani, H. Sorkhi et al., "COVID-19 and digestive system in children: a retrospective study," Archives of Iranian Medicine, vol. 23, no. 11, pp. 782-786, 2020.

[27] M. Samy and L. M. Khalaf, "Chest CT features of COVID-19 pediatric patients presented with upper respiratory symptoms," Egyptian Journal of Radiology and Nuclear Medicine, vol. 52, no. 1, pp. 1-5, 2021.

[28] D. Sun, H. Li, X.-X. Lu et al., "Clinical features of severe pediatric patients with coronavirus disease 2019 in Wuhan: a single center's observational study," World Journal of Pediatrics, vol. 16, p. 1, 2020.

[29] C. Zhang, J. Gu, Q. Chen et al., "Clinical and epidemiological characteristics of pediatric SARS-CoV-2 infections in China: a multicenter case series," PLoS Medicine, vol. 17, no. 6, Article ID e1003130, 2020. 